\title{
Has U.S. Inflation Really Become Harder to Forecast?
}

\section{Lanne, Markku}

2012

Lanne , M \& Luoto , J 2012 , ' Has U.S. Inflation Really Become Harder to Forecast? ' ,

Economics Letters, vol. 115 , no. 3 , pp. 383-386 . https://doi.org/10.1016/j.econlet.2011.12.088

http://hdl.handle.net/10138/230229

https://doi.org/10.1016/j.econlet.2011.12.088

cc_by_nc_nd

acceptedVersion

Downloaded from Helda, University of Helsinki institutional repository.

This is an electronic reprint of the original article.

This reprint may differ from the original in pagination and typographic detail.

Please cite the original version. 
This is the peer reviewed version of the following article: Lanne, Markku, and Luoto, Jani (2012): Has US inflation really become harder to forecast? Economics Letters, 115, 383 - 386, which has been published in final form at https://doi.org/10.1016/j.econlet.2011.12.088. (C) 2018. This manuscript bersion is made available under the CC-BY-NC-ND 4.0 license http://creativecommons.org/licenses/by-nc-nd/4.0/

\title{
Has U.S. Inflation Really Become Harder to Forecast?
}

\author{
Markku Lanne ${ }^{\mathrm{a}, \mathrm{b}, 1}$, Jani Luoto ${ }^{\mathrm{a}, \mathrm{b}}$ \\ ${ }^{a}$ Department of Political and Economic Studies, University of Helsinki, Finland \\ ${ }^{b}$ HECER, University of Helsinki
}

\begin{abstract}
Since the mid-1980s, Phillips curve forecasts of U.S. inflation have been inferior to those of a conventional causal autoregression. However, little change in forecast accuracy is detected against the benchmark of a noncausal autoregression, more accurately characterizing U.S. inflation dynamics.
\end{abstract}

Key words: Inflation forecast, Noncausal time series, Phillips curve JEL: classification C22, C53, E31

\section{Introduction}

In their recent, widely cited article, Stock and Watson (2007, SW henceforth) argued that while U.S. inflation in general has become easier to forecast after 1983, it has also become more difficult to improve upon univariate models by means of backward-looking Phillips curve (PC) forecasts. Specifically, they claim that before 1983, PC models were superior to the univariate autoregressive (AR) model, but after 1984, the situation has reversed. We argue that SW's benchmark model is not the appropriate univariate model, especially in the 1970-1983 period, but, in fact, inflation dynamics are better captured by a noncausal, instead of a conventional causal AR model. This claim is backed up by the findings of Lanne and Saikkonen (2011) and Lanne et al. (2009) for the CPI inflation and Lanne et al. (2011) for the GDP price inflation. Also, in contrast to SW, we do not force a unit root in the inflation process.

\footnotetext{
${ }^{1}$ Corresponding author. Department of Political and Economic Studies, P.O. Box 17 (Arkadiankatu 7), 00014 University of Helsinki, Finland. Tel.: +358 9191 28731; fax: +358 9191 28736. E-mail address: markku.lanne@helsinki.fi.
} 
Our results show that once the noncausal AR benchmark is adopted, the changes in the forecastability of U.S. GDP inflation are minor, and mainly confined to the two-year forecast horizon. As to the other inflation measures (personal consumption expenditure deflator for core items (PCE-core) and all items (PCE-all), and the consumer price index (CPI-U)) considered by $\mathrm{SW}$, the $\mathrm{PC}$ forecasts very rarely beat the noncausal AR forecast in either forecast period.

The plan of the paper is as follows. In Section 2, we present the noncausal AR model, and discuss estimation and forecasting. Section 3 presents the forecasting results and comparisons to SW's findings. Finally, Section 4 concludes.

\section{Noncausal AR Model}

Let us consider the following noncausal AR model for inflation $\pi_{t}(t=0, \pm 1, \pm 2, \ldots)$,

$$
\varphi\left(B^{-1}\right) \phi(B) \pi_{t}=\epsilon_{t}
$$

where $\phi(B)=1-\phi_{1} B-\cdots-\phi_{r} B^{r}, \varphi\left(B^{-1}\right)=1-\varphi_{1} B^{-1}-\cdots-\varphi_{s} B^{-s}$, and $\epsilon_{t}$ is a sequence of independent, identically distributed (continuous) random variables with mean zero and variance $\sigma^{2}$ or, briefly, $\epsilon_{t} \sim$ i.i.d. $\left(0, \sigma^{2}\right)$. Moreover, $B$ is the usual backward shift operator, that is, $B^{k} \pi_{t}=\pi_{t-k}$ $(k=0, \pm 1, \ldots)$, and the polynomials $\phi(z)$ and $\varphi(z)$ have their zeros outside the unit circle so that

$$
\phi(z) \neq 0 \text { for }|z| \leq 1 \text { and } \varphi(z) \neq 0 \text { for }|z| \leq 1 .
$$

This formulation was recently suggested by Lanne and Saikkonen (2011). We use the abbreviation $\operatorname{AR}(r, s)$ for the model defined by (1). If $\varphi_{1}=\cdots=$ $\varphi_{s}=0$, model (1) reduces to the conventional causal $\operatorname{AR}(r)$ model.

The conditions in (2) imply that $\pi_{t}$ has the two-sided moving average representation

$$
\pi_{t}=\sum_{j=-\infty}^{\infty} \psi_{j} \epsilon_{t-j},
$$

where $\psi_{j}$ is the coefficient of $z^{j}$ in the Laurent series expansion of $\phi(z)^{-1} \varphi\left(z^{-1}\right)^{-1} \stackrel{\text { def }}{=}$ $\psi(z)$. Note that this implies that past observations can be used to predict future errors. From (1) one also obtains the representation

$$
\pi_{t}=\phi_{1} \pi_{t-1}+\cdots+\phi_{r} \pi_{t-r}+v_{t},
$$


where $v_{t}=\varphi\left(B^{-1}\right)^{-1} \epsilon_{t}=\sum_{j=0}^{\infty} \beta_{j} \epsilon_{t+j}$ with $\beta_{j}$ the coefficient of $z^{j}$ in the power series expansion of $\varphi\left(B^{-1}\right)^{-1}$. This representation can be used to obtain forecasts. Taking conditional expectations conditional on past and present inflation of (4) yields

$$
\pi_{t}=\phi_{1} \pi_{t-1}+\cdots+\phi_{r} \pi_{t-r}+E_{t}\left(\sum_{j=0}^{\infty} \beta_{j} \epsilon_{t+j}\right),
$$

which shows that in a noncausal AR model, future errors are predictable by past values of inflation. If the true model is noncausal, but this is ignored in forecasting, i.e., forecasts are based on a causal AR model, this predictability is dismissed, leading to inferior forecast accuracy despite the causal and noncausal forecasts being based on the same information.

A well-known feature of noncausal autoregressions is that a non-Gaussian error term is required to achieve identification (see, e.g., Breidt et al., 1991, and Brockwell and Davis, 1987, p. 124-125). This follows from the fact that the same autocovariance function can be obtained irrespective of whether the roots of $\phi(z)$ and $\varphi(z)$ in (1) are inside or outside the unit circle, i.e., whether $\pi_{t}$ is causal or noncausal. Since the Gaussian likelihood is completely determined by the autocovariance function, causal and noncausal processes cannot be distinguished under Gaussianity. Therefore, following Lanne and Saikkonen (2011), we specify Student's t-distribution for $\epsilon_{t}$. In addition to these authors, also Lanne et al. $(2009,2011)$ have shown this distribution to fit U.S. inflation series well. A small value of the degrees-of-freedom parameter is required for identification, as otherwise the t-distibution comes close to the normal distribution, and identification is not achieved (or it is weak). ${ }^{2}$ Under this assumption, the noncausal AR model can be estimated by maximizing the approximate likelihood function proposed by Lanne and Saikkonen (2011). The approximation involves conditioning on the first $r$ and last $s$ observations. As the orders of the polynomials are typically small, the approximation error is likely to be negligible.

To compute forecasts based on representation (4), simulation methods are called for. Let $E_{T}(\cdot)$ signify the conditional expectation operator given the observed data vector $\boldsymbol{\pi}=\left(\pi_{1}, \ldots, \pi_{T}\right)$. From (4) it is seen that the optimal

\footnotetext{
${ }^{2}$ For the inflation series considered in Section 3, the degrees-of-freedom parameter is estimated small, indicating strong identification. For instance, for the GDP price inflation series, the estimate for the entire sample period is 4.94 with a standard error of 1.82 .
} 
predictor of $\pi_{T+h}(h>0)$ based on $\pi$ satisfies

$$
E_{T}\left(\pi_{T+h}\right)=\phi_{1} E_{T}\left(\pi_{T+h-1}\right)+\cdots+\phi_{r} E_{T}\left(\pi_{T+h-r}\right)+E_{T}\left(v_{T+h}\right) .
$$

Thus, if we are able to forecast the variable $v_{T+h}$, we can compute forecasts of inflation recursively. In the purely noncausal case of particular interest in this paper, the optimal forecast of $\pi_{T+h}$ reduces to $E_{T}\left(v_{T+h}\right)$. To calculate $v_{T+h}$ in practice we use the approximation $v_{T+h} \approx \sum_{j=0}^{M-h} \beta_{j} \epsilon_{T+h+j}$, where the integer $M$ is supposed to be so large that the approximation error is negligible for all forecast horizons $h$ of interest. To a close approximation we then have

$$
E_{T}\left(\pi_{T+h}\right) \approx \phi_{1} E_{T}\left(\pi_{T+h-1}\right)+\cdots+\phi_{r} E_{T}\left(\pi_{T+h-r}\right)+E_{T}\left(\sum_{j=0}^{M-h} \beta_{j} \epsilon_{T+h+j}\right) .
$$

Lanne et al. (2011) show how to generate by simulation the conditional density of future errors needed in the computation of the conditional expectation of $\sum_{j=0}^{M-h} \beta_{j} \epsilon_{T+h+j}$. Following their recommendations based on simulation experiments, we set $M=50$, and the number of replications, $N$, in the simulation procedure equals 100000 .

\section{Forecast Results}

We focus on quarterly GDP price index inflation, but we also considered a number of other inflation measures (PCE-core, PCE-all and CPI-U). ${ }^{3}$ All data are downloaded from Mark Watson's web page. The PC forecasts are calculated using autoregressive distributed lag models with various activity variables and potentially gap variables based on them as additional regressors (SW's equation (3)). The specifications PC- $\Delta \mathrm{u}, \mathrm{PC}-\Delta \mathrm{y}, \mathrm{PC}-\Delta \mathrm{CapUtil}$ and PC- $\Delta$ Permits omit gap variables. For detailed variable definitions, see SW.

The noncausal AR models are estimated recursively, with data from 1960:I-1969:IV used for initial parameter estimation. Following SW, forecast results are presented separately for the periods 1970:I-1983:IV and 1984:I2004:IV. Unlike SW, we only consider iterated multistep forecasts that SW

\footnotetext{
${ }^{3}$ To save space, the results are not reported, but they are available upon request. The general conclusion are the same as those for GDP price inflation.
} 
found quantitatively quite similar to their direct forecasts. Lanne and Saikkonen (2011) propose a model selection procedure that was employed in forecasting by Lanne et al. (2011). However, in this paper all noncausal forecasts are based on the recursively estimated fixed $\operatorname{AR}(0,4)$ model that should be adequate for quarterly data. SW mainly rely on the Akaike information criterion (AIC) in model selection, i.e., they recursively select the order of the AR model (denoted AR(AIC) below). However, they also show that the fixed $\operatorname{AR}(4,0)$ model produces similar results.

Table 1 reproduces the root mean squared forecast errors (RMSFE) of the $\mathrm{AR}(\mathrm{AIC})$ forecast and the relative mean squred forecast errors (MSFE) of a number of alternative models in relation to that model from SW's Tables 1 and 4. Compared to the benchmark AR(AIC) model, the predictive performance of virtually all PC models is inferior in the latter compared to the former subsample period at all horizons. This is even more clearly seen in the left panel of Table 3 that presents the percentage changes of the relative MSFEs. There are only two negative entries, both of which are small in absolute value compared to the positive percentage changes. Moreover, while in the 1970-1983 period, the relative MSFEs in Table 1 are, in general, less than unity, indicating the superiority of the PC models, the situation is reversed in the 1984-2004 period. This evidence warrants SW's claim that since the mid-1980s, it has been difficult for inflation forecasts to improve on univariate models.

If the purely noncausal $\mathrm{AR}(0,4)$ model is used as the benchmark, the results are drastically different. As the figures in Table 2 show, the PC forecasts are, in general inferior to this univariate benchmark model. In contrast to Table 1 , this is the case also in the 1970-1983 period, while the performance of the $\mathrm{AR}(\mathrm{AIC})$ and $\mathrm{AR}(0,4)$ models is similar in the 1984-2004 period. As a result, the changes in predictive accuracy of the $\mathrm{PC}$ models are, in general, much smaller than SW's results in Table 1 lead one to believe. Moreover, the right panel of Table 3 shows that in many cases, the predictive performance of these models has improved, especially at horizons of four quarters or less, and in case of relative deterioration, it is much lesser than suggested by SW. Particularly noteworthy is the result that the model with the change in building permits as the predictor (PC- $\Delta$ Permits) is the only model that beats the $\operatorname{AR}(0,4)$ benchmark at all horizons in the latter subsample period, and shows great improvement in predictive accuracy over the 1970-1983 period.

In addition to the $\mathrm{PC}$ forecasts, SW also considered the random walk forecasts (AO) of Atkeson and Ohanian (2001). In accordance with their 
results, we also find that these forecasts improve upon the $\mathrm{AR}(0,4)$ and $\mathrm{PC}$ forecasts at the one- and two-year horizons in the 1984-2004 period but not at other horizons or in the first subsample period.

Although the differences in predictive accuracy of the noncausal AR model are smaller than those of the causal AR(AIC) model, also the RMSFEs of the former have declined since 1984. Hence, in accordance with the findings of SW, in absolute terms, inflation has become easier to forecast even when the set of univariate models is extended by the noncausal AR model.

\section{Conclusion}

We have shown that compared to the noncausal AR benchmark model, U.S. inflation has hardly become more difficult to forecast by means of backward-looking PC models since the mid-1980s, contrary to the claims of SW, who used the causal autoregression as the benchmark. At the very least the differences are much smaller than SW found. Based on the findings in the previous literature, U.S. inflation dynamics are better described by a noncausal than a causal AR model, and hence, the noncausal model should be taken as the benchmark model against which the PC forecasts are judged. These findings are reinforced by the fact that the noncausal $\operatorname{AR}(0,4)$ model also consistently produces more accurate forecasts than the causal $\operatorname{AR}(4,0)$ or AR(AIC) models in the 1970-1983 period and has comparable accuracy in the 1984-2004 sample. Our results show, that compared to this univariate benchmark, the PC models provide poor forecasts both before and after the mid-1980s.

Acknowledgement 1. Financial support from the Academy of Finland is gratefully acknowledged.

\section{References}

[1] Atkeson, A., Ohanian, L.E., 2001. Are Phillips curves useful for forecasting inflation? Federal Reserve Bank of Minneapolis Quarterly Review, $25: 1,2-11$.

[2] Breidt, F.J., Davis, R.A., Lii, K.-S., Rosenblatt, M., 1991. Maximum likelihood estimation for noncausal autoregressive processes. Journal of Multivariate Analysis 36, 175-198. 
[3] Brockwell, P.J., Davis, R.A., 1987. Time Series: Theory and Methods. Springer-Verlag. New York.

[4] Lanne, M., Saikkonen, P., 2011. Noncausal autoregressions for economic time series. Journal of Time Series Econometrics 3 (3), Article 2.

[5] Lanne, M., Luoma, A., Luoto, J., 2009. Bayesian model selection and forecasting in noncausal autoregressive models. Journal of Applied Econometrics (forthcoming).

[6] Lanne, M., Luoto, J., Saikkonen, P., 2011. Optimal forecasting of noncausal autoregressive time series. International Journal of Forecasting (forthocming).

[7] Stock, J.H., Watson, M.W., 2007. Why has U.S. inflation become harder to forecast? Journal of Money, Credit and Banking, Supplement to Vol. $39,3-33$. 
Table 1: Pseudo out-of-sample forecasting results for GDP inflation with the AR(AIC) model as the benchmark.

\begin{tabular}{|c|c|c|c|c|c|c|c|c|}
\hline & \multicolumn{4}{|c|}{ 1970:I-1983:IV } & \multicolumn{4}{|c|}{ 1984:I-2004:IV } \\
\hline & $h=1$ & $h=2$ & $h=4$ & $h=8$ & $h=1$ & $h=2$ & $h=4$ & $h=8$ \\
\hline AR(AIC) RMSFE & 1.72 & 1.75 & 1.89 & 2.38 & 0.78 & 0.68 & 0.62 & 0.73 \\
\hline \multicolumn{9}{|l|}{ Relative MSFEs } \\
\hline $\mathrm{AR}(\mathrm{AIC})$ & 1.00 & 1.00 & 1.00 & 1.00 & 1.00 & 1.00 & 1.00 & 1.00 \\
\hline $\mathrm{AO}$ & 1.95 & 1.57 & 1.06 & 1.00 & 1.22 & 1.10 & 0.89 & 0.84 \\
\hline $\mathrm{PC}-u$ & 0.85 & 0.92 & 0.88 & 0.61 & 0.95 & 1.11 & 1.48 & 1.78 \\
\hline $\mathrm{PC}-\Delta u$ & 0.87 & 0.87 & 0.86 & 0.64 & 1.06 & 1.27 & 1.83 & 2.21 \\
\hline PC-ugap ${ }^{1-s i d e d}$ & 0.88 & 0.99 & 0.98 & 0.87 & 1.06 & 1.29 & 1.84 & 2.39 \\
\hline $\mathrm{PC}-\Delta y$ & 0.99 & 1.06 & 0.93 & 0.58 & 1.05 & 1.06 & 1.23 & 1.53 \\
\hline PC-ygap ${ }^{1-s i d e d}$ & 0.94 & 0.97 & 0.99 & 0.78 & 0.97 & 0.97 & 1.25 & 1.55 \\
\hline PC-CapUtil & 0.85 & 0.88 & 0.79 & 0.55 & 0.95 & 1.01 & 1.35 & 1.52 \\
\hline PC- $\Delta$ CapUtil & 1.02 & 1.00 & 0.87 & 0.64 & 1.03 & 1.10 & 1.30 & 1.51 \\
\hline PC-Permits & 0.93 & 1.02 & 0.98 & 0.78 & 1.08 & 1.23 & 1.31 & 1.52 \\
\hline PC- $\Delta$ Permits & 1.02 & 1.04 & 0.99 & 0.86 & 1.00 & 1.00 & 1.00 & 1.02 \\
\hline $\operatorname{AR}(4,0)$ & 0.95 & 1.08 & 1.05 & 0.93 & 0.93 & 0.96 & 0.99 & 0.94 \\
\hline $\operatorname{AR}(0,4)$ & 0.87 & 0.81 & 0.75 & 0.66 & 1.03 & 1.02 & 1.01 & 1.07 \\
\hline
\end{tabular}

The table reproduces results in Stock and Watson's (2007) Tables 1 and 4 and presents our results based on the noncausal $\operatorname{AR}(0,4)$ model. The first row reports the root mean squared forecast errors of the causal $\mathrm{AR}(\mathrm{AIC})$ benchmark forecast. The rest of the entries are the relative mean squared forecast errors relative to the AR(AIC) benchmark. 
Table 2: Pseudo out-of-sample forecasting results for GDP inflation with the $\operatorname{AR}(0,4)$ model as the benchmark.

\begin{tabular}{|c|c|c|c|c|c|c|c|c|}
\hline & \multicolumn{4}{|c|}{ 1970:I-1983:IV } & \multicolumn{4}{|c|}{ 1984:I-2004:IV } \\
\hline & $h=1$ & $h=2$ & $h=4$ & $h=8$ & $h=1$ & $h=2$ & $h=4$ & $h=8$ \\
\hline $\operatorname{AR}(0,4) \mathrm{RMSFE}$ & 1.60 & 1.58 & 1.64 & 1.93 & 0.79 & 0.69 & 0.62 & 0.76 \\
\hline \multicolumn{9}{|l|}{ Relative MSFEs } \\
\hline $\operatorname{AR}(0,4)$ & 1.00 & 1.00 & 1.00 & 1.00 & 1.00 & 1.00 & 1.00 & 1.00 \\
\hline $\mathrm{AO}$ & 2.25 & 1.93 & 1.41 & 1.51 & 1.19 & 1.08 & 0.88 & 0.78 \\
\hline $\mathrm{PC}-u$ & 0.98 & 1.14 & 1.18 & 0.93 & 0.92 & 1.09 & 1.46 & 1.66 \\
\hline $\mathrm{PC}-\Delta u$ & 1.00 & 1.07 & 1.15 & 0.96 & 1.03 & 1.25 & 1.82 & 2.06 \\
\hline PC-ugap $p^{1-s i d e d}$ & 1.01 & 1.21 & 1.31 & 1.32 & 1.04 & 1.27 & 1.82 & 2.23 \\
\hline $\mathrm{PC}-\Delta y$ & 1.14 & 1.30 & 1.25 & 0.88 & 1.02 & 1.04 & 1.22 & 1.43 \\
\hline PC-ygap ${ }^{1-s i d e d}$ & 1.09 & 1.20 & 1.33 & 1.18 & 0.94 & 0.96 & 1.24 & 1.44 \\
\hline PC-CapUtil & 0.98 & 1.08 & 1.06 & 0.84 & 0.93 & 0.99 & 1.34 & 1.41 \\
\hline PC- $\Delta$ CapUtil & 1.18 & 1.23 & 1.16 & 0.97 & 1.00 & 1.08 & 1.29 & 1.41 \\
\hline PC-Permits & 1.08 & 1.25 & 1.31 & 1.19 & 1.05 & 1.21 & 1.29 & 1.42 \\
\hline PC- $\Delta$ Permits & 1.18 & 1.28 & 1.32 & 1.30 & 0.97 & 0.98 & 0.99 & 0.95 \\
\hline $\operatorname{AR}(4,0)$ & 1.10 & 1.33 & 1.34 & 1.51 & 0.97 & 0.98 & 0.99 & 0.93 \\
\hline
\end{tabular}

The first row reports the root mean squared forecast errors of the $\operatorname{AR}(0,4)$ benchmark forecast. The rest of the entries are the relative mean squared forecast errors relative to the $\operatorname{AR}(0,4)$ benchmark. 
Table 3: Percentage changes in the relative MSFE in relation to the AR(AIC) model (left panel) and the AR(0,4) model (right panel) between the 1970-1983 and 1984-2004 periods.

\begin{tabular}{|c|c|c|c|c|c|c|c|c|}
\hline & \multicolumn{8}{|c|}{ Benchmark Model } \\
\hline & \multicolumn{4}{|c|}{ AR(AIC) } & \multicolumn{4}{|c|}{$\operatorname{AR}(0,4)$} \\
\hline & $h=1$ & $h=2$ & $h=4$ & $h=8$ & $h=1$ & $h=2$ & $h=4$ & $h=8$ \\
\hline $\mathrm{AO}$ & $-46.3 \%$ & $-35.7 \%$ & $-17.6 \%$ & $-17.0 \%$ & $-63.5 \%$ & $-57.8 \%$ & $-47.8 \%$ & $-65.4 \%$ \\
\hline $\mathrm{PC}$ & & 18 & 51.6 & 106. & $-5.8 \%$ & $-4.1 \%$ & & 58.3 \\
\hline & $20.2 \%$ & $38.3 \%$ & $76.3 \%$ & 124.2 & $3.1 \%$ & $16.2 \%$ & $46.1 \%$ & $75.8 \%$ \\
\hline PC- & $19.4 \%$ & $26.6 \%$ & $63.0 \%$ & $100.6 \%$ & $2.2 \%$ & $4.4 \%$ & $32.8 \%$ & $52.2 \%$ \\
\hline PC- $\Delta$ & $6.1 \%$ & $0.3 \%$ & $27.6 \%$ & $96.4 \%$ & $-11.0 \%$ & $-21.9 \%$ & $-2.6 \%$ & $48.0 \%$ \\
\hline PC-y & $2.7 \%$ & $0.0 \%$ & $23.8 \%$ & $68.1 \%$ & $-14.5 \%$ & $-22.2 \%$ & $-6.4 \%$ & $19.7 \%$ \\
\hline PC-Cap & $11.9 \%$ & $13.4 \%$ & $53.2 \%$ & $100.8 \%$ & $-5.3 \%$ & $-8.8 \%$ & $23.0 \%$ & $52.4 \%$ \\
\hline $\mathrm{PC}-\Delta \mathrm{C}$ & $0.0 \%$ & $9.5 \%$ & $40.2 \%$ & $86.3 \%$ & $-17.1 \%$ & $-12.7 \%$ & $10.0 \%$ & $37.9 \%$ \\
\hline PC-Pe & $14.6 \%$ & $18.5 \%$ & $28.6 \%$ & $66.3 \%$ & $-2.5 \%$ & $-3.7 \%$ & $-1.6 \%$ & $17.9 \%$ \\
\hline & $-2.4 \%$ & $-4.3 \%$ & $1.5 \%$ & $16.9 \%$ & $-19.5 \%$ & $-26.5 \%$ & $-28.7 \%$ & $-31.5 \%$ \\
\hline
\end{tabular}

